\title{
Human platelet 12-lipoxygenase: Naturally occurring Q261/R261 variants and N544L mutant show altered activity but unaffected substrate binding and membrane association behavior
}

\author{
ANSARI M. ALEEM ${ }^{1,5}$, LEIGH WELLS ${ }^{1,6}$, JERZY JANKUN ${ }^{1,2}$, MATTHIAS WALTHER $^{3}$, \\ HARTMUT KÜHN $^{3}$, JEANNETTE REINARTZ ${ }^{4}$ and EWA SKRZYPCZAK-JANKUN ${ }^{1}$ \\ ${ }^{1}$ Urology Research Center, College of Medicine, University of Toledo, 3000 Arlington Ave., \\ Toledo, OH 43614, USA; ${ }^{2}$ Medical Academy of Gdansk, Department of Clinical Nutrition, \\ Debinki 7, 80-211 Gdansk, Poland; ${ }^{3}$ University Medicine Berlin - Charité, Monbijoustrasse 2, \\ D-10117 Berlin; ${ }^{4}$ American Diagnostica GmbH, Kaplaneigasse 35, D-64319 Pfungstadt, Germany
}

Received August 21, 2009; Accepted September 21, 2009

DOI: 10.3892/ijmm_00000289

\begin{abstract}
The single nucleotide polymorphism (SNP) R261Q in the human platelet 12-lipoxygenase has been correlated with several human diseases. To understand better the biological performance we have compared enzymatic properties of the recombinant enzymes: 'wild-type' as Q261 and R261 variants with a single Q261R mutation at the enzyme periphery and N544L mutant with an altered active site. The R261 variant does not follow the same kinetics such as WT-Q261 showing a lag phase, a slower accumulation of product, following a different time-course without reaching plateau characteristic for the Q261 variant. The N544L substitution in the active site almost eradicates enzymatic activity proving that asparagine is as important for catalysis as the conserved histidines and C-terminal isoleucine. All three enzymes have comparable
\end{abstract}

Correspondence to: Dr Ewa Skrzypczak-Jankun, The University of Toledo - HSC, College of Medicine, Department of Urology, Mail Stop 1091, 3000 Arlington Ave., Toledo, OH 43614, USA

E-mail: ewa.skrzypczak-jankun@utoledo.edu

Present address: ${ }^{5}$ University Diabetes Center, King Saud University, Riyadh, KSA;

${ }^{6}$ Des Moines University, College of Osteopathic Medicine, 3200 Grand Avenue, Des Moines, IA 50312, USA

Abbreviations: AA, arachidonic acid; hp-12LOX, human platelet 12(S)-lipoxygenase; 5LOX, 5-lipoxygenase; 12R-LOX, 12(R)-lipoxygenase, 15LOX-1 and 15LOX-2, 15-lipoxygenase type 1 and 2; eLOX3, epidermal lipoxygenase-3; COX-1 and COX-2, cyclooxygenase-1 and -2; 12(S)HETE, 12S-hydroxyeicosatetraenoic acid; MALDI-TOF, matrix-assisted laser desorption ionization time-offlight; MAPK, mitogen-activated protein kinase; MW, molecular weight; SNP, single nucleotide polymorphism; WT, wild-type

Key words: 12-lipoxygenase, single nucleotide polymorphism, SNP R261Q, arachidonic acid binding, membrane binding substrate binding and membrane association behavior. We conclude that the naturally occurring SNP, causing single mutation at a location distant to the active site, can alter the protein-protein association of this oligomeric enzyme making impact on kinetic properties of an allosteric mechanism and molecular recognition/signaling at a submembrane frontier.

\section{Introduction}

Human platelet 12-lipoxygenase (hp-12LOX, SwissProt P18054, 663 residues + Fe non-heme cofactor), implicated in lipid signaling, is present mainly in cytoplasm of the various cell types and occurs in nature as several variants: E259K, Q261R, A298T, N322S, R430H. In addition to an arachidonate activity leading to 12(S)HETE it exhibits a hepoxylinsynthase activity, which involves the intermediate formation of an epoxide. Its biological relevance is also related to its interactions with other proteins, such as integrins, keratin type II cytoskeletal 5, lamin-A/C, protein kinase $\mathrm{C}$ and other MAPK kinases. By participating in biosynthesis of eicosanoids it affects many biological processes such as positive regulation of cell motility, cell adhesion, cell growth and cell proliferation. It has also been associated with molecular and cell signaling and its activity can be regulated by glutathione $(1,2)$. In our studies of the recombinant enzyme we have used 'wild-type' (WT) designation for the sequence with amino acids shown above in bold. Recent cohort studies tracing single nucleotide polymorphism (SNP) in the hp-12LOX gene brought to attention a possible connection between the presence of a given SNP and the susceptibility of its carriers to the certain ailments. According to a molecular structure [PDB:3d31, (3)] the sequential substitutions in the four variants of hp-12LOX (except $\mathrm{R} 430 \mathrm{H}$ which is in a distant location from the others) are concentrated in a fragment (residues $220-330$ ), which forms a distinguish subdomain on the outskirt of a catalytic moiety of the enzyme (4). Each of them provides changes in the local charge, size and shape of the amino acids. Tracking the data on the Q261R variants one can find evidence that it 
makes a statistically significant presence in some diseases. According to clinical studies Gln/Gln genotype prevails in patients with esophageal and colorectal cancer, diabetic nephropathy, bipolar disorder and seems to sway negative regulation of bone mineral density (5-11). While the Arg/Arg genotype was observed more often (46\%) than Arg/Gln (42\%) and Gln/Gln (12\%) among patients with essential hypertension and correlated with significantly higher secretion of urinary 12(S)HETE in GG homozygous individuals (12). The available data for SNP coding position 261 in hp-12LOX could be very controversial. Tan et al published statistics for 1,000 colorectal cancer patients and 1,300 controls showing positive association of hp-12LOX and COX-2 polymorphism with this disease (8). Goodman et al evaluating 293 patients and corresponding controls found a statistically significant association of colon cancer with 5LOX (with differences depending on racial factor), but not with $12 \mathrm{LOX}, \mathrm{COX}-1$ or COX-2 (13). Gong et al reported positive association for 12LOX, but not 5LOX in 162 cases with 211 controls (6). Thus even for the same disease, the results of such studies seem to depend on the size and ethnicity of the cohort and might have been impacted by the tumor characteristics. Study of esophageal squamous cell carcinoma (ESCC) was the only case were platelets of healthy volunteers where genotyped and examined for 12LOX activity, which for calculated average was at 3-fold higher level for Gln/Gln vs. Arg/Arg homozygous individuals (7).

Human platelet 12-lipoxygenase is acting at the dynamic frontier of biological membranes where molecules balance between compartmentalization and communication in transport and signaling processes. Studies on biological samples could be 'tainted' by the presence of other molecules. To exclude these secondary effects and to investigate the impact of amino acid exchanges on the properties of enzyme we have expressed hp-12LOX variants and characterized the resulting enzyme species: WT (containing Q261), Q261R (imitating natural SNP), as well as WT-N544L mutant, which served as an example of the alteration done in the vicinity of iron cofactor vs. the peripheral location (Fig. 1).

\section{Materials and methods}

Expression of the recombinant enzymes in Sf9 system, chromatographic purification, activity, for the $\mathrm{N}_{\mathrm{t}}$-6His-tag recombinant proteins were carried out side-by-side, following the protocols described (1). All proteins were aliquoted and stored in $0.1 \mathrm{M}$ Tris $\mathrm{HCl}, 0.1 \mathrm{M} \mathrm{KCl}, \mathrm{pH} 8.0$ and samples from the same purification batch were used for all analyses. The reaction time course of enzymatic activity was monitored continuously at $234 \mathrm{~nm}$ using Beckman Coultier DU640B UV-VIS spectrometer as change in absorbance caused by conjugated dienes [molar absorption coefficient $\varepsilon=27 \mathrm{mM}^{-1}$ $\mathrm{cm}^{-1}$ for $\left.12 \mathrm{HpETE}(14)\right]$ in $1 \mathrm{ml}$ cuvette containing $1 \mathrm{ml}$ solution with $20 \mathrm{mM}$ Hepes- $\mathrm{NaOH} \mathrm{pH} 8.0,33 \mu \mathrm{M}$ arachidonic acid to which $10 \mu \mathrm{g}$ of enzyme was added.

Iron content was determined 40 days after purification by Inductively Coupled Plasma (ICP) spectrometry (University of California, Santa Cruz, CA - courtesy of Dr T. Holman) using an internal Co standard and external standardized $\mathrm{Fe}$ solutions.

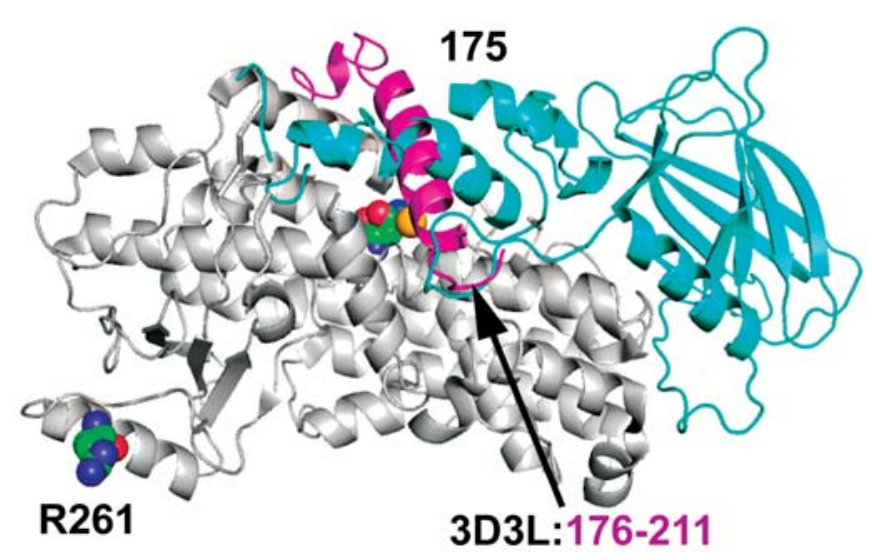

Figure 1. Structure of human platelet 12-lipoxygenase: Gray - a ribbon representation of PDB entry 3D3L depicting residues 176-662, with 176-211 in purple, marking the difference from the homology model. Cyan - missing fragments taken from the homology modeling (1). Orange ball represents $\mathrm{Fe}$-cofactor, green, blue and red balls show C, N, O atoms from residues N544 and R261.

Arachidonic acid (AA) binding was examined by MALDITOF mass spectrometry (Alphalyse Inc., Palo Alto, CA). The proteins were analyzed twice by MALDI mass spectrometry in linear mode using sinapinic acid as matrix. The instrument was tested to give best resolution at the $75 \mathrm{kDa}$ range. The mass spectra were calibrated using an external calibration. The proteins were desalted using Millipore biomax $30 \mathrm{~K}$ centrifugal filters. For arachidonate binding analysis $2 \mu \mathrm{l}$ of arachidonic acid were added and incubated for $30 \mathrm{~min}$. A laser beam was directed at the dry matrix, molecules vaporized and the ionized proteins were measured in a time-of-flight (TOF) mass analyzer.

Membrane-binding assay. Endoplasmic membrane preparations $(2 \mu \mathrm{l})$ were incubated at room temperature for $10 \mathrm{~min}$ with $2.5 \mu \mathrm{g}$ of previously centrifuged $(20,000 \mathrm{x} \mathrm{g}, 15 \mathrm{~min}$, $4^{\circ} \mathrm{C}$ ) enzyme in $50 \mathrm{mM}$ Hepes $\mathrm{pH} 7.4$, containing $150 \mathrm{mM}$ $\mathrm{NaCl}$ and $1 \mathrm{mM} \mathrm{Ca}$ (total assay volume $25 \mu \mathrm{l}$ ) and processed following the protocol given previously (1).

Comparison of human lipoxygenases by the native-PAGE. This non-denaturating method was used to compare all human lipoxygenases utilizing a kit from Invitrogen containing Novex ${ }^{\circledR}$ Tris-Glycine Native Running Buffer (10X): used at 1x, Novex ${ }^{\circledR}$ Tris-Glycine Native Sample Buffer $(2 \mathrm{X})$ : used at $1 \mathrm{x}$ and containing $10 \mathrm{mM}$ DTT. Protein $(500 \mathrm{ng})(0.2-0.3 \mu \mathrm{l})$ in a volume of $12 \mu \mathrm{l}$ of the sample buffer/DTT was applied per lane of the $4-12 \%$ Tris Glycin gels. The recombinant enzymes were the generous gifts of Dr T. Holman, University of California at Santa Cruz, CA (5LOX, 15LOX-1 and 15LOX-2) and Dr A. Brash, Vanderbilt University Medical Center, Nashville, TN (12R-LOX, eLOX3).

\section{Results}

The three expressed proteins were $\sim 95 \%$ pure and were analyzed simultaneously 'side-by-side' for better comparison.

SNP Q261 and R261 variants differ in kinetics. The enzymatic activity evaluated by $12 \mathrm{HETE}$ formation approaches 3,800 and 

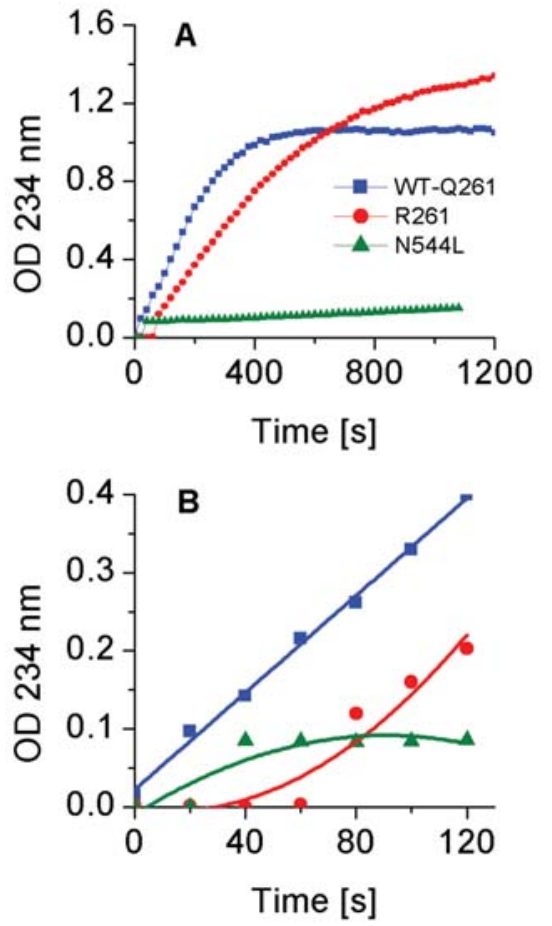

Figure 2. Time-course of the lipoxygenase activity (OD234 nm vs. time, $10 \mu \mathrm{g}$ of protein in $1 \mathrm{ml}$ sample, $33 \mu \mathrm{M}$ arachidonic acid) for the wild-type variants and N544L mutant: (A) All data normalized to the same iron content as in WT-Q261. (B) The enlargement showing the polynomial trend lines during the first $120 \mathrm{sec}$.

Table I. Membrane binding for hp-12LOX mutants.

\begin{tabular}{lccc}
\hline Sample: & WT S/P & Q261R S/P & N544L S/P \\
\hline \% of enzyme in & $24 / 76$ & $5 / 95$ & $6 / 94$ \\
supernatant (S) & $39 / 61$ & $10 / 90$ & $16 / 84$ \\
in pellet (P) & $4 / 96$ & $4 / 96$ & $4 / 96$ \\
Average & $22 / 78$ & $6 / 94$ & $9 / 91$ \\
\pm SD & \pm 18 & \pm 3 & \pm 6 \\
p (WT vs. mutant) & & 0.18 & 0.27 \\
\hline
\end{tabular}

P, represents 100,000 g pellet; S, 100,000 g supernatant.

2,500 $\mathrm{nmol} / \mathrm{mg}$ at $10 \mathrm{~min}$ accumulation for Q261 and R261 variants respectively, while only $250 \mathrm{nmol} / \mathrm{mg}$ for $\mathrm{N} 544 \mathrm{~L}$ mutant in the freshly purified samples at $22^{\circ} \mathrm{C}$, for data not normalized for the iron content. The average standardized count for Fe in the investigated samples was $34 \times 10^{4}$ for WT, $23 \times 10^{4}$ for Q261R and $24 \times 10^{4}$ for WT-N544L mutant with an error $\pm 3 \times 10^{4}$ counts for the Fe standard. The data measured in triplicate were consistent and $>3$ times higher than the fluctuations observed for the standard indicating that the differences in the iron content were real and corresponded to 30 and $26 \%$ less iron in the Q261R and N544L mutants, respectively. Fig. $2 \mathrm{~A}$ and $\mathrm{B}$ showing data normalized to the same iron content for all samples provide evidence that judging activity by $12 \mathrm{HETE}$ accumulation at some arbitrary chosen point of time with the iron content as an unknown variable
Table II. Mass spectrometry (MALDI-TOF) results without and in the presence of arachidonic acid (all $\mathrm{MW}$ in $\mathrm{kDa}$, estimated error \pm 200 ).

\begin{tabular}{|c|c|c|c|c|}
\hline \multirow[b]{2}{*}{ Sample } & \multirow[b]{2}{*}{ MW calc ${ }^{\mathrm{a}}$} & \multicolumn{2}{|c|}{$\mathrm{m} / \mathrm{z}:^{+}$obs } & \multirow[b]{2}{*}{$\Delta \mathrm{MW}$ obs } \\
\hline & & no AA & $+\mathrm{AA}^{\mathrm{b}}$ & \\
\hline WT Q261 & 76573 & 76462 & 77597 & 1135 \\
\hline Q261R & 76601 & 76607 & 77606 & 999 \\
\hline N544L & 76572 & 76386 & 77606 & 1220 \\
\hline
\end{tabular}

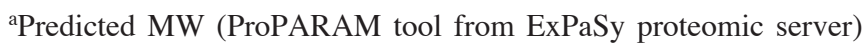
including 6His-tag and Fe-cofactor. ${ }^{\mathrm{b}} \mathrm{AA}$ arachidonic acid MW 304.5.

could be misleading. The R261 variant shows a lag phase and much slower product accumulation at the start, but can outperform WT-Q261 later on. Our measurements of activity vs. time at various concentrations of arachidonic acid (from 0.23 to $99 \mu \mathrm{M}$ ) revealed a lag phase of $10-30 \mathrm{sec}$ at every concentration of fatty acid (data not shown) and the obtained data did not allow us to explain the kinetics in a concise mathematical fashion. It is clear that SNP variant R261 does not follow the equation describing kinetic performance of its WT-Q261 counterpart (1).

N544L mutation in the active site diminishes activity to the level of only several percent of the one exhibited by the WT-enzyme. This significant drop in activity is unrelated to the iron content and has to be attributed to the change in the enzyme local structure introduced by the replacement of a polar Asn in the vicinity of the iron cofactor by a similar in size but hydrophobic and apolar Leu.

Membrane and substrate binding are not affected by Q261R and N544L mutations. In the membrane binding assay done in triplicate the percentage distribution of enzymes between supernatant (S) and membrane pellets (P) shows wider discrepancy between WT samples 22/78 18 and more consistent results for Q261R and N544L yet comparable for all three proteins within the experimental error (Table I). MALDI-TOF mass spectrometry done in duplicate (average results given in Table II) showed prominent peaks at $\sim 76 \mathrm{kDa}$ for WT, Q261R and WT-N544L with increase in $\mathrm{m} / \mathrm{z}$ by $\geq 1,000 \mathrm{kDa}$ when preincubated with arachidonic acid. Taking into account that at this mass an estimated error is $\sim 200 \mathrm{kDa}$ the change observed may correspond to at least 2 but more likely 3 molecules of fatty acid per one $\mathrm{MH}^{+}$ion of enzyme.

Recombinant human lipoxygenases are stable as oligomers in the solution. We have used the native-PAGE method to catch a glimpse of more realistic, 'native' state of the human lipoxygenases in the solution (Fig. 3). 5-LOX was not highly purified and thus is omitted as inconclusive. All lipoxygenases have similar MW 75-80 kDa and based on homology and molecular modeling are believed to have similar topology. Under our experimental conditions 15-LOX-2 aligns with 12S-LOX which has been proven to be stable as a dimer (1), 


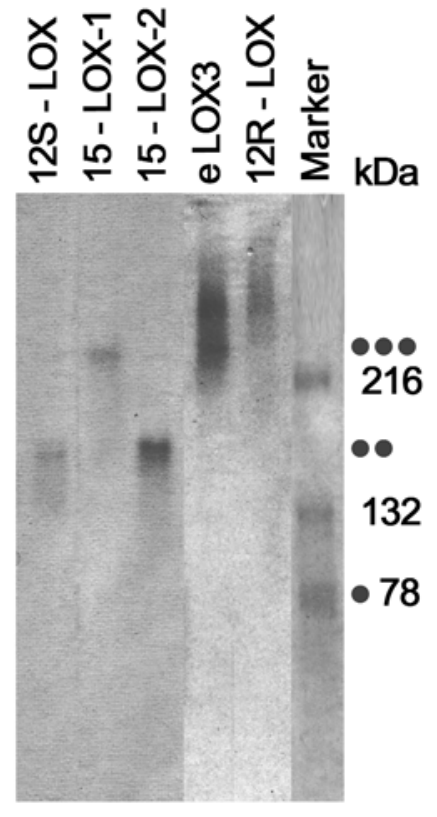

Figure 3. Native-PAGE of human lipoxygenases (MW in $\mathrm{kDa}$ as in SwissProt without $0.9 \mathrm{kDa}$ for $6 \mathrm{His}$ and iron): 12S-lipoxygenase (P18054, 75.7), 15-lipoxygenase-1 (P16050, 74.7), 15-lipoxygenase-2 (O15296, 75.9), epidermis-type lipoxygenase-3 (Q9BYJ1, 80.5) and 12R-lipoxygenase (O75342, 80.4). The dots mark approximate position expected for monomer, dimer and trimer.

15-LOX-1 position appears higher suggesting a trimer, while eLOX3 and 12R-LOX might be trimers or higher oligomers.

\section{Discussion}

The enzymatic activity (Fig. 2) is clearly different for the recombinant SNP variants, but the observed discrepancy is lower than cited for WT variants obtained from humans. For enzymes isolated from human plasma Guo et al reported $0.405 \pm 0.047$ and $0.136 \pm 0.022 \mathrm{nmol} / \mathrm{mg} / \mathrm{min}$ activity of $\mathrm{hp}-$ 12LOX for individuals with GG and AA alleles respectively, i.e. 3 times higher for variant with Q261 (7). In studies of Lagarde et al this parameter for human platelets was at 3-4 $\mathrm{nmol} / \mathrm{mg} / \mathrm{min}$ level or $20 \mathrm{nmol} / \mathrm{mg}$ of protein at $10 \mathrm{~min}$ accumulation (15). Hp-12LOX activity diminishes very quickly with time and strongly depends on the environmental factors such as temperature, buffer, $\mathrm{pH}$, amount of substrate and other additives (salts, reducing agents, surfactants) $(1,14,16)$. Since the conditions in different experiments were not standardized the reported numbers greatly vary and cannot be compared directly (Table III). Also, the purity of the sample and the iron content have a very strong impact on the activity. Unfortunately, in the majority of publications the enzyme:iron stoichiometry is not addressed but most likely never equimolar. Thus, when corrected to $1: 1$ the kinetic values may appear much higher (16) than observed otherwise. For 12-lipoxygenases the iron, when measured, was reported in the range 0.01 to 0.67 of iron per one molecule of enzyme, with the values for the native sequence usually higher than for the various mutants from the same series of experiments $(1,16-18)$. There is always a question about compatibility when comparison is made between native and recombinant protein. Chen and Funk (19) observed lower
Table III. Literature data concerning activity of human 12lipoxygenase.

\begin{tabular}{lccc}
\hline & $\begin{array}{c}\text { Activity } \\
\text { Source of 12LOX }\end{array}$ & & \\
\hline Human (blood) & & & \\
Platelets & $300 \mathrm{C})$ & Ref. \\
Platelets & $3.0-4.0$ & 37 & $(20)$ \\
& $17-20(10 \mathrm{~min})^{\mathrm{a}}$ & & $(15)$ \\
Platelets: Arg/Arg & $0.06-0.22$ & & $(15)$ \\
R261Q Arg/Gln & $0.04-0.62$ & & $(7)$ \\
variants Gln/Gln & $0.2-0.6$ & & $(7)$ \\
H. Recombinant & & & \\
HEK293 native & $97.6(10 \mathrm{~min})^{\mathrm{b}}$ & & $(19)$ \\
with 6His-Ntag & $75.0(10 \mathrm{~min})^{\mathrm{b}}$ & & $(19)$ \\
H/Q at Fe-site & 0 & & $(19)$ \\
Sf9 & $2000-4000$ & 37 & $(14)$ \\
Sf9 & $6250^{\mathrm{c}}$ & 22 & $(16)$ \\
Sf9 Q261 variant & $3800(10 \mathrm{~min})^{\mathrm{a}, \mathrm{d}}$ & 22 \\
R261 variant & $2500(10 \mathrm{~min})^{\mathrm{a}, \mathrm{d}}$ & 22 \\
N544L & $250(10 \mathrm{~min})^{\mathrm{a}, \mathrm{d}}$ & 22 & \\
& &
\end{tabular}

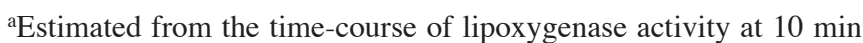
accumulation. ${ }^{\mathrm{b}}$ Activity in $10,000 \mathrm{~g}$ supernatant from transfected HEK293 cells at $10 \mathrm{~min}$. ${ }^{\mathrm{c} E s t i m a t e d ~ u s i n g ~} \mathrm{k}_{\text {cat }}=8$ at $22^{\circ} \mathrm{C}$, iron content corrected to $1 .{ }^{\mathrm{d}} \mathrm{Data}$ not normalized for iron content.

activity $(77 \%)$ for the 6 His-tag-12LOX vs. native, while review of available data shows in general higher numbers for recombinant, His-tagged enzymes than for native extracted from platelets (Table III). It is noteworthy that in contrast to Hada et al (20) Guo et al and Lagarde et al $(7,15)$ used Tyrode buffer which prevents platelets aggregation, but it contains carbonates that were found to promote iron withdrawal in creating apo-enzyme from soybean lipoxygenase (21).

The conclusion from our experiment is that the activity observed for the recombinant variant Q261R shows a change less dramatic than 3-fold reported for the average values observed for 12LOX variants from platelets of healthy, human volunteers with GG $(n=6)$ and AA $(n=10)$ genotypes $[\mathrm{p}=0.001$, (7)]. The recorded activities may represent variations in the iron content in lipoxygenase from different individuals. This parameter was not determined and a larger pool of Gln/Arg heterozygotes $(n=22)$ shows numbers covering both ranges of the other two groups. However, a multivariate logistic regression model applied to cancer patients and controls shows increased risk of developing ESCC for individuals with GG allele over AA homozygotes (odds ratio 1.42, 95\% confidence interval 1.12-1.81, $\mathrm{p}=0.003$, with $1.08,0.88-1.32$ and $\mathrm{p}=0.466$ for GA). While the iron deficiency in $\mathrm{hp}$ 12LOX (22) may be a real cause for imparied activity a single mutation in a location crucial for the enzymatic performance could have a more serious impact (Table III). 
In relation to the WT protein and its Q261R variant, the WT-N544L mutant, for which the amino acid defining the active site was exchanged, exhibited only a marginal catalytic activity. This was irrelevant to the iron presence since Q261R had a similar content. Previous studies concerning active site of mammalian $12 \mathrm{LOX}$ were focused on substituting the iron ligands bound to the central metal ion, such as histidines (19) or the C-terminal isoleucine (23). N544 in hp-12LOX might be at a longer distance but geometrically in a proper place of the iron's coordination polyhedron, and participating in a tightly woven hydrogen bonding network, like in soybean enzymes (24) where the role of this residue was extensively studied $(25,26)$. Human lipoxygenases 5-, 12S- 12R- and eLOX3 have Asn while 15LOX has His in type 1 and Ser in type 2, which might contribute to their so remarkably different behavior $(27,28)$. We do not know a high resolution structure of any human lipoxygenase except an engineered catalytic domain of hp12LOX depicted on Fig. 1, corresponding to residues 172662 in sequence and representing variant R261 (PDB:3d31) (3). In that structure Asn544 has its side chain $\sim 6 \AA$ away from $\mathrm{Fe}$ but participates in a strong hydrogen bonding network (Fe-water...Asn544...Gln547...Glu356... His360), which could be preserved in the intact enzyme and essential for interactions with the substrate, like in soy LOXs. Our study proves that Asn544 is as important for catalysis as the residues bound to iron (19).

The Q261R variant has an undisturbed active site, with mutation at a great distance, but this mutation can affect how molecules associate into oligomers and thus alter active site accessibility or the way how the enzyme performs its allosteric mechanism. Although PDB:3d31 structure might not depict truly the WT enzyme due to the engineering done, the location of R261 is far away from the parts that might be disturbed by the expression vectors. Thus a portrayal of this residue

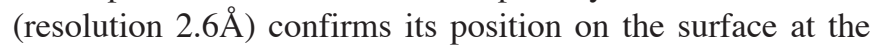
outskirt of the molecule and pointing outward (Fig. 1). The replacement $\mathrm{Q} \leftrightarrow \mathrm{R}$ affects all crucial elements $\left(\mathrm{pK}_{\mathrm{a}}\right.$, size and shape) of molecular interactions and surface complementarity so it might be important in molecular recognition and complex formation (4), consequently influencing a supramolecular chemistry of the interlocked molecular architecture in the biological system.

To investigate how the mutations Q261R and N544L can affect arachidonic acid processing all three expressed enzymes were tested for membrane and substrate binding. The WT exhibits less adhesion to the membranes (Table I), but considering a wide margin of error observed for this assay this difference cannot be claimed as significant. Also, our experiment provides evidence that enzymatic activity is not required for membrane binding as was suggested (29). Although results from MALDI-TOF (Table II) vary for the individual mutants it cannot be said based on these data that the mutants behave differently than WT enzyme regarding arachidonic acid binding.

Our understanding of the precise functionality of the molecules at the submembrane location in the biological environment is daunting. Recently a concept has been developed that 'enzyme mechanisms should be viewed as catalytic networks with multiple conformations that occur serially and in parallel mechanism' (30). It has been found that hp-12LOX enzymatic activity strongly depends on self-association into aggregates with higher oligomers being more active than dimers (1). Comparison of human lipoxygenases using native-PAGE to show their 'native' state in solution indicates that formation of stable oligomers may be true for other human lipoxygenases as well (Fig. 3). They all could be allosteric although their kinetic properties might be different $(28,31,32)$. Bearing that in mind and the fact that each molecule of hp-12LOX can bind to 3 molecules of substrate/product one can presume that any changes in self-association would impinge on the enzymatic activity.

Our conclusions from this study are: i) Asn544 is crucial for catalysis. This single mutation N544L practically inactivates the enzyme probably by disrupting the local chain of interactions (hydrogen bonds) surrounding the active site. ii) The enzymatic activity is not required for membrane binding and for the inactive enzyme or one with diminished activity could be on par with or higher than for the active one. iii) Single nucleotide polymorphism causing Q261R variation in sequence imposes changes in enzymatic activity without a significant impact on its ability for membrane or arachidonic acid binding. The differences in biological activity could be a consequence of how single mutation in the molecular structure changes behavior of this enzyme at the submembrane frontier in vivo, making impact on the diseases stimulated by this lipoxygenase.

\section{Acknowledgements}

E.S.J. thanks Dr T. Holman for help with ICP-MS and discussions. We thank Dr R. Hart, American Diagnostica, Inc. and Frank D. Stranahan Endowment Fund for Oncological Research for financial support.

\section{References}

1. Aleem AM, Jankun J, Dignam JD, et al: Human platelet 12lipoxygenase, new findings about its activity, membrane binding and low-resolution structure. J Mol Biol 376: 193-209, 2008.

2. Canals S, Casarejos MJ, de Bernardo S, Rodriguez-Martin E and Mena MA: Nitric oxide triggers the toxicity due to glutathione depletion in midbrain cultures through 12-lipoxygenase. J Biol Chem 278: 21542-21549, 2003.

3. Tresaugues L, Moche M, Arrowsmith, $\mathrm{CH}$, Berglund $\mathrm{H}$, Busam RD, Collins R, Dahlgren LG, Edwards AM, Flodin S, Flores A, Graslund S, Hammarstrom M, Herman MD, Johansson A, Johansson I, Kallas A, Karlberg T, Kotenyova T, Lehtio L, Nilsson ME, Nyman T, Olesen K, Persson C, Sagemark J, Schueler H, Svensson L, Thorsell AG, Van Den Berg S, Welin M, Weigelt $J$, Wikstrom $M$ and Nordlund P: Crystal structure of the lipoxygenase domain of human arachidonate 12-lipoxygenase, 12s-type (In press), PDB entry 3D3L, 2008. http://www.pdb.org/pdb/explore/explore.do? structureld $=3 \mathrm{D} 3 \mathrm{~L}$

4. Jankun J, Doerks T, Aleem AM, Lysiak-Szydlowska W and Skrzypczak-Jankun E: Do human lipoxygenases have a pdz regulatory domain? Curr Mol Med 8: 768-773, 2008.

5. Liu Y, Freedman BI, Burdon KP, et al: Association of arachidonate 12-lipoxygenase genotype variation and glycemic control with albuminuria in type 2 diabetes. Am J Kidney Dis 52: 242-250, 2008.

6. Gong Z, Hebert JR, Bostick RM, et al: Common polymorphisms in 5-lipoxygenase and 12-lipoxygenase genes and the risk of incident, sporadic colorectal adenoma. Cancer 109: 849-857, 2007. 
7. Guo Y, Zhang X, Tan W, et al: Platelet 12-lipoxygenase arg $261 \mathrm{gln}$ polymorphism: Functional characterization and association with risk of esophageal squamous cell carcinoma in combination with cox-2 polymorphisms. Pharmacogenet Genomics 17: 197-205, 2007.

8. Tan W, Wu J, Zhang X, et al: Associations of functional polymorphisms in cyclooxygenase-2 and platelet 12-lipoxygenase with risk of occurrence and advanced disease status of colorectal cancer. Carcinogenesis 28: 1197-1201, 2007.

9. Fridman C, Ojopi EP, Gregorio SP, et al: Association of a new polymorphism in alox12 gene with bipolar disorder. Eur Arch Psychiatry Clin Neurosci 253: 40-43, 2003.

10. Mullin BH, Spector TD, Curtis CC, et al: Polymorphisms in alox12, but not alox 15, are significantly associated with bmd in postmenopausal women. Calcif Tissue Int 81: 10-17, 2007.

11. Ichikawa S, Koller DL, Johnson ML, et al: Human alox12, but not alox 15 , is associated with bmd in white men and women. J Bone Miner Res 21: 556-564, 2006.

12. Quintana LF, Guzman B, Collado S, Claria J and Poch E: A coding polymorphism in the 12-lipoxygenase gene is associated to essential hypertension and urinary 12(s)-hete. Kidney Int 69 : 526-530, 2006.

13. Goodman JE, Bowman ED, Chanock SJ, Alberg AJ and Harris CC: Arachidonate lipoxygenase (alox) and cyclooxygenase (cox) polymorphisms and colon cancer risk. Carcinogenesis 25: 2467-2472, 2004.

14. Chen XS, Brash AR and Funk CD: Purification and characterization of recombinant histidine-tagged human platelet 12-lipoxygenase expressed in a baculovirus/insect cell system. Eur J Biochem 214: 845-852, 1993.

15. Lagarde M, Croset M, Authi KS and Crawford N: Subcellular localization and some properties of lipoxygenase activity in human blood platelets. Biochem J 222: 495-500, 1984.

16. Segraves EN and Holman TR: Kinetic investigations of the ratelimiting step in human 12- and 15-lipoxygenase. Biochemistry 42: 5236-5243, 2003.

17. Suzuki H, Kishimoto K, Yoshimoto T, et al: Site-directed mutagenesis studies on the iron-binding domain and the determinant for the substrate oxygenation site of porcine leukocyte arachidonate 12-lipoxygenase. Biochim Biophys Acta 1210: 308-316, 1994

18. Deschamps JD, Gautschi JT, Whitman S, et al: Discovery of platelet-type 12-human lipoxygenase selective inhibitors by high-throughput screening of structurally diverse libraries. Bioorg Med Chem 15: 6900-6908, 2007.

19. Chen XS and Funk CD: Structure-function properties of human platelet 12-lipoxygenase: Chimeric enzyme and in vitro mutagenesis studies. FASEB J 7: 694-701, 1993.
20. Hada T, Ueda N, Takahashi Y and Yamamoto S: Catalytic properties of human platelet 12-lipoxygenase as compared with the enzymes of other origins. Biochim Biophys Acta 1083: 89-93, 1991.

21. Kariapper MS, Dunham WR and Funk MO Jr: Iron extraction from soybean lipoxygenase 3 and reconstitution of catalytic activity from the apoenzyme. Biochem Biophys Res Commun 284: 563-567, 2001.

22. Tomo K, Takayama H, Kaneko Y, et al: Qualitative platelet 12-lipoxygenase abnormality in a patient with essential thrombocythemia. Thromb Haemost 77: 294-297, 1997.

23. Chen XS, Kurre U, Jenkins NA, Copeland NG and Funk CD Cdna cloning, expression, mutagenesis of c-terminal isoleucine, genomic structure, and chromosomal localizations of murine 12-lipoxygenases. J Biol Chem 269: 13979-13987, 1994.

24. Berman HM, Westbrook J, Feng Z, et al: The protein data bank. Nucleic Acids Res 28: 235-242, 2000

25. Kramer JA, Johnson KR, Dunham WR, Sands RH and Funk MO Jr: Position 713 is critical for catalysis but not iron binding in soybean lipoxygenase 3. Biochemistry 33: 15017$15022,1994$.

26. Segraves EN, Chruszcz M, Neidig ML, et al: Kinetic, spectroscopic, and structural investigations of the soybean lipoxygenase-1 first-coordination sphere mutant, asn694gly. Biochemistry 45: 10233-10242, 2006.

27. Shureiqi I and Lippman SM: Lipoxygenase modulation to reverse carcinogenesis. Cancer Res 61: 6307-6312, 2001.

28. Wecksler AT, Jacquot C, van der Donk WA and Holman TR: Mechanistic investigations of human reticulocyte 15- and platelet 12-lipoxygenases with arachidonic acid. Biochemistry 48: 6259-6267, 2009.

29. van Leyen K, Duvoisin RM, Engelhardt H and Wiedmann M: A function for lipoxygenase in programmed organelle degradation. Nature 395: 392-395, 1998.

30. Benkovic SJ, Hammes GG and Hammes-Schiffer S: Freeenergy landscape of enzyme catalysis. Biochemistry 47: 3317-3321, 2008

31. Kharytonenko HI, Skaterna TD, Mel'nyk AK, Babii LV and Kharchenko OV: Interaction between 5-lipoxygenase and allosteric effector - sodium dodecyl sulfate. Ukr Biokhim Zh 80: 31-39, 2008.

32. Wecksler AT, Kenyon V, Garcia NK, Deschamps JD, van der Donk WA and Holman TR: Kinetic and structural investigations of the allosteric site in human epithelial 15lipoxygenase-2. Biochemistry 48: 8721-8730, 2009. 